\title{
DETERMINANTES DE LA DESNUTRICIÓN CRÓNICA DE LOS MENORES DE TRES AÑOS EN LAS REGIONES DEL PERÚ: SUB-ANÁLISIS DE LA ENCUESTA ENDES 2000
}

\author{
DETERMINANTS OF STUNTING IN CHILDREN LESS THAN THREE YEARS IN THE \\ REGIONS OF PERU: SUB-ANALYSIS OF THE SURVEY ENDES 2000
}

1*Luz Bullón C. y ${ }^{2}$ Laura Astete R.

\begin{abstract}
Resumen
El objetivo fue identificar los determinantes más importantes de la desnutrición crónica en niños menores de tres años de edad de las diferentes regiones del Perú, según la información recopilada en la Encuesta de Demográfica y de Salud Familiar (ENDES) 2000 del Instituto Nacional de Estadística e Informática (INEI). Se realizó un sub-análisis utilizando datos de la ENDES 2000, y no otras que tienen datos más actuales, debido al tamaño y diseño muestral. Se seleccionaron las variables significativas que entraron en el modelo multivariado (análisis bivariados, con una significancia de 10\%). Los sujetos analizados fueron menores de tres años, según los datos recogidos en la ENDES 2000. La prevalencia de retraso de crecimiento en niños menores de tres años es el 21,7\%. Los determinantes son diferentes en cada región natural, y los más importantes en todos los ámbitos fueron: educación de la madre, controles de crecimiento de los niños, número de controles pre- natales, lugar del parto, peso al nacer del niño y el número de hijos vivos de la madre. Se concluye que la desnutrición crónica en niños menores de tres años tiene particularidades en cada región, por lo que es importante plantear la resolución este problema nutricional de diferentes maneras. Adicional a las acciones actuales, es necesario incluir mejoras en la condición de la mujer.
\end{abstract}

Palabras Clave: Determinantes de la desnutrición, la desnutrición crónica, retraso de crecimiento, estado nutricional.

\begin{abstract}
The objective was to find determinants of stunting in under-three age children in different regions of Peru, on the basis of the Population and Family Health Survey (ENDES) 2000 by the National Institute of Statistics and Information (INEI). A sub-analysis using data from the ENDES 2000 was performed because of the sample methods and size, than more updated ENDES' version. It was selected significant variables for the multivariate model (Bivariate analyses, significance was 10\%).Subject analyzed were children under the age of three, by data extracted from the ENDES 2000. The prevalence of stunting in under-three children is $21.7 \%$. The determinants are different in each natural region, and the most important factors in all regions were: mother's education, growth's control of child, number of pre-natal controls, place of delivery, child's birthweight and number of living children. It was concluded that stunting in underthree children has particularities in each area, so it is important to address this nutritional problem in different ways. Additional to current actions, is needed to include improvements in women status.
\end{abstract}

Key words: Stunting determinants, chronic malnutrition, stunting, nutritional status.

\section{Introducción}

En el Perú la desnutrición crónica afecta a 17.9\% (según patrón NCHS) de los niños y niñas menores de cinco años (INEI, 2011) y desde hace diez años se ha mantenido en cifras similares (INEI, 1996 e INEI, 2000). Los análisis estadísticos se hicieron entre los años 2006 al 2008, en un contexto de cambio de patrón de referencia para evaluar la desnutrición infantil, por tanto, todos los análisis del presente estudio son hechos con el patrón de referencia NCHS.

El objetivo de la presente investigación es identificar los determinantes más importantes de la desnutrición crónica en menores de tres años en las regiones del Perú, según la

\footnotetext{
Departamento Académico de Estadística e Informática, Facultad de Economía y Planificación, Universidad Nacional Agraria La Molina, Apartado Postal 12-056 - La Molina, Lima (Perú). Email: luz@lamolina.edu.pe.

${ }^{2}$ Maestría de Nutrición Pública, Escuela de Posgrado, Universidad Nacional Agraria La Molina

${ }^{3}$ NCHS son las iniciales de National Center for Health Statistics, y es el patrón de referencia de crecimiento infantil muy utilizado hasta hace unos años atrás, pues actualmente se utiliza el patrón de referencia de la Organización Mundial de la Salud (OMS) publicado en el 2006 y difundido en nuestro país más ampliamente a partir del 2008-2009. La desnutrición crónica afecta al 23.2\% de los menores de 5 años según el patrón de referencia OMS (INEI, 2011).
} 
información recopilada en la Encuesta Demográfica y de Salud Familiar (ENDES) 2000.

La malnutrición ${ }^{4}$ es un problema multicausal y complejo (UNICEF, 1998) y (ACC/SCN, 2000). En América Latina la desnutrición crónica es más notoria en países como Guatemala, Honduras, Haití, Ecuador, Bolivia y Perú (SCN, 2005), afectando en este último al 17.9\% (INEI, 2011) de la población infantil. Un niño desnutrido tiene menor capacidad inmunológica, por tanto, mayor riesgo de padecer enfermedades diarreicas (Brown, 2003) y respiratorias, de morir por estas causas (Rice et al, 2001). La desnutrición afecta también el desarrollo de los niños, comprometiendo su rendimiento escolar (Jukes et al, 2002), especialmente, mientras más severa es la desnutrición (Méndez y Adair, 1999).

\subsection{La encuesta demográfica y de salud familiar (ENDES)}

La ENDES es realizada por el Instituto Nacional de Estadística e Informática (INEI) en el marco de las Encuestas de Demografía y Salud, (Demographic and Health Statistic, DHS). En el Perú se han realizado las ENDES desde 1986, y a partir del 2004-2005, la denominada ENDES Continua, que se recoge cada año, y cada 5 años completa la muestra. Con la ENDES se ha podido obtener información a nivel nacional, acerca de la fecundidad, mortalidad y en general de la salud de las mujeres en edad fértil y los niños. La ENDES tiene como objetivo general proveer de información actualizada del Perú y sus departamentos, sobre la dinámica demográfica y el estado de salud de las madres y los niños menores de cinco años.

Se ha propuesto analizar las determinantes de la desnutrición crónica en los niños menores de tres años en las diferentes regiones naturales del Perú a partir de la información de la ENDES 2000. Debido al tamaño y diseño muestral, la ENDES 2000 es la que puede ser utilizada para el tipo de análisis estadísticos y el nivel de inferencia que se presentan a continuación. Datos más actuales provenientes de la ENDES, no posibilitarían tal desagregación.

\subsection{Causas de la desnutrición: marco conceptual de UNICEF}

Según el marco conceptual de la UNICEF (UNICEF, 1998), se tiene:

\subsubsection{Causas inmediatas de la desnutrición}

Dentro de las causas inmediatas están consumo inadecuado de nutrientes (Onyango et al, 1998), como la ausencia de adecuados lactancia materna y alimentación complementaria; y las enfermedades infecciosas, que son las que afectan además el consumo y utilización de los nutrientes. Se sabe que la lactancia materna exclusiva durante los primeros seis meses de vida, con extensión a los 24 meses junto a otros alimentos, es lo mejor para el crecimiento del niño (Fewtrell et al, 2005), (Thi Hip et al, 2000) y (Marquis et al, 1997); reduce la mortalidad por infecciones respiratorias y diarreicas, incluso en lactantes que viven en ambientes desfavorables (Villapando et al, 2000) (Arifeen y Black, 2001). El efecto de la diarrea

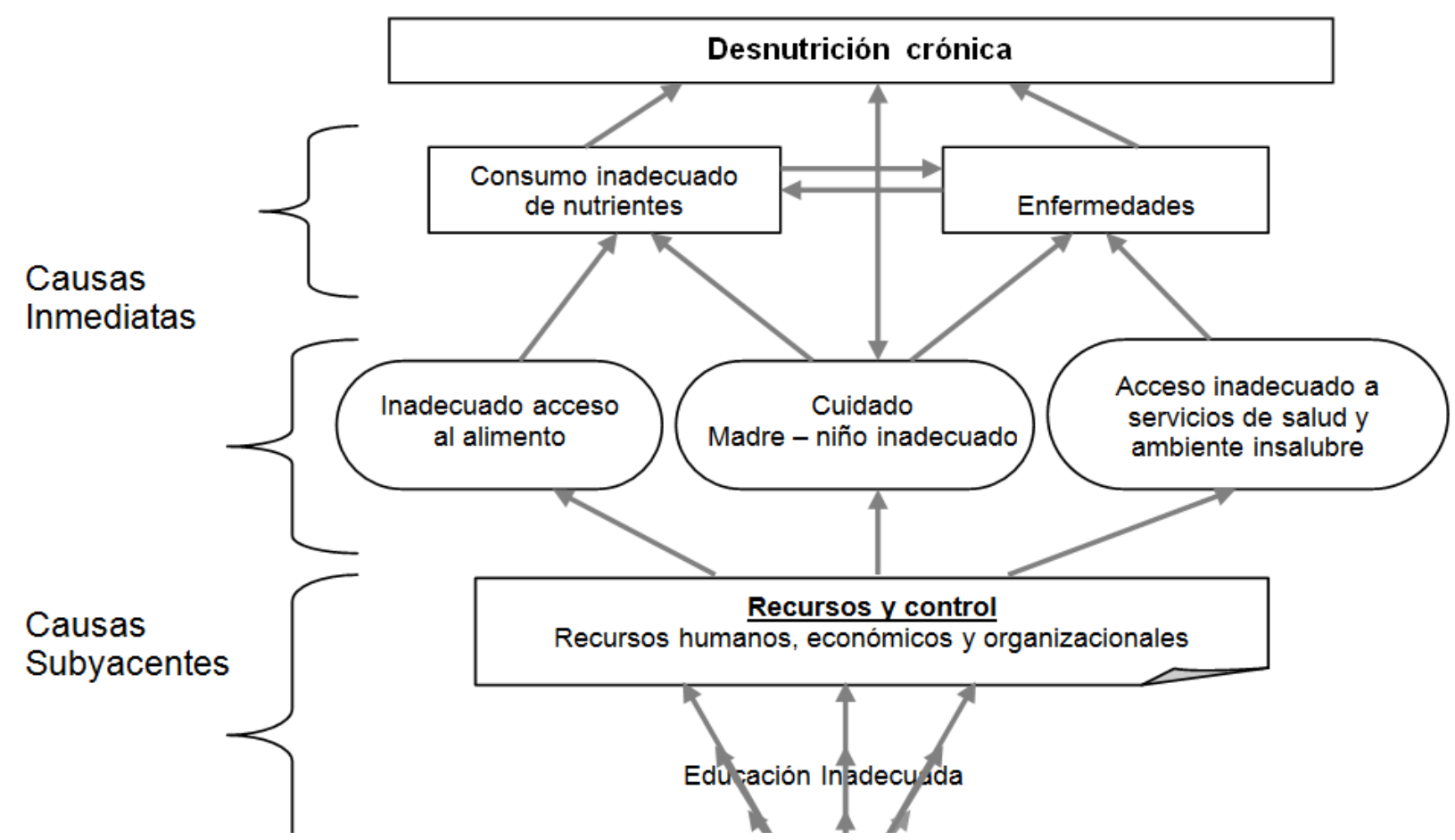

Figura 1. Marco conceptual de la desnutrición crónica (UNICEF, 1998)

${ }^{4}$ Malnutrición es el término comúnmente utilizado para describir los problemas de déficit, así como los de exceso. 
y malnutrición es bidireccional (Brown, 2003), pues la diarrea perjudica el estado nutricional, y los niños desnutridos padecen más episodios de diarrea. Bajo ingesta inadecuada, la diarrea afecta el crecimiento lineal, (Stephensen, 1999), (VanDerslice et al, 1994) y (Lutter, et al, 1992). La relación más fuerte y consistente encontrada entre la desnutrición y la mortalidad infantil ha sido observada para infecciones respiratorias y diarrea (Assis et al, 2005) y (De Caballero et al, 2004).

\subsubsection{Causas subyacentes de la desnutrición}

Las causas subyacentes tienen que ver con el hogar, dentro de los cuales figuran saneamiento y servicios de salud, acceso a alimentos, y el cuidado madre- niño. Una investigación transversal que utilizó encuestas DHS de sesenta y tres países se identificó como los determinantes subyacentes más importantes además de la educación de la mujer, el estado de la mujer relativo al varón, la disponibilidad per capita de alimento y el saneamiento (Smith y Haddad, 1990).

El grado de educación en la madre o cuidadora es determinante del estado nutricional (Delpeuch et al, 2000), (Bhandari y Smith, 2000) del niño, aunque el efecto de la educación de la madre está muy influenciado por el nivel socioeconómico (Bellessa, 2005).

En zonas con peores condiciones de agua y saneamiento se puede observar que los niños presentan una talla más baja y más episodios de diarrea que los que tienen mejores condiciones (Checkley, 2004) y (Merchant et al, 2003). Los servicios de salud preventivos (Ruel et al, 1999) y las inmunizaciones (Ukwuani y Suchindran, 2003) han demostrado ser determinantes fuertes para un buen estado nutricional del niño, incluso en madres con poca educación y pertenecientes a hogares con escasos recursos económicos (UNICEF, 2005).

\subsubsection{Causas básicas de la desnutrición}

Por último, tenemos como las causas básicas de desnutrición a la estructura política y económica, el ambiente sociocultural y los recursos potenciales (ambiente, tecnología, personas), la pobreza (UNICEF, 1998) y (SCN, 2005). También encontramos factores como la ocupación del jefe del hogar (Reyes et al, 2004), la etnicidad indígena (Larrea, et al, 2004), cambios estacionales (Brown et al, 1982), ingreso nacional per capita y la democracia (Smith, et al 2002), entre otros.

\subsection{Estudio previo sobre determinantes de la desnutrición}

Un análisis de las determinantes de la desnutrición crónica y aguda en el Perú (Suárez et al, 1999) encontró que la desnutrición aguda tuvo asociación con muy pocas de las determinantes seleccionadas, a diferencia de la desnutrición crónica que mostró tener relación con diversas variables estudiadas, como grado de instrucción del jefe de familia y de la madre, control prenatal de la madre, lugar del parto, peso al nacer, sexo del niño, número de hijos vivos, prevalencia de diarrea, material del piso y el tipo de servicios higiénicos. En el grupo de menores de seis meses las determinantes de la desnutrición crónica más importantes fueron los relacionados al acceso a los servicios de salud; mientras que en los mayores de seis meses las variables socioeconómicas fueron más relevantes (INEI y Prisma, 1999).

\section{Materiales y métodos}

El diseño de estudio es analítico y transversal. Es un subanálisis que se hace a partir de la ENDES 2000 recogida por el INEI, luego de haber pasado por un proceso de re-codificación de variables y limpieza. Por su diseño y tamaño muestral, se puede hacer inferencia en el ámbito nacional, por lugar de residencia (urbano/ rural) y por regiones naturales, incluso por departamentos. Los resultados en el presente estudio se presentan en los diferentes ámbitos ${ }^{5}$, que son: Lima Metropolitana, Resto de Costa, Sierra Urbana, Sierra Rural y Selva. Por otro lado, se separó a la Sierra según lugar de residencia para que el análisis sea más enriquecedor.

Se incluye sólo a los niños menores de 3 años, porque es importante conocer las determinantes de la desnutrición crónica en quienes son intervenidos en los proyectos y programas sociales.

La muestra original de la ENDES 2000 es probabilística, autoponderada por departamentos y áreas, estratificada, multietápica e independiente para cada departamento. En la ENDES 2000 se tomó la talla a 10477 niños menores de 5 años (INEI, 2001). Tomando sólo a los niños menores de 3 años (criterio de inclusión), la muestra final para el presente análisis es 6070 niños menores de 3 años, y excluyendo cualquier situación patológica.

Las bases de datos otorgadas fueron recodificadas o recategorizadas a nuevas variables que fueron definidas en base al modelo de UNICEF (1998). Como este estudio utiliza una fuente de información secundaria, no se ha planificado qué indicadores recoger, sino que se seleccionaron y construyeron los que fueron necesarios para que formen parte de los factores asociados de la desnutrición según el marco conceptual de UNICEF. El listado de las variables se puede observar en la Tabla $\mathrm{N}^{\circ} 1$, mientras que una descripción más detallada de las mismas en la $\mathrm{N}^{\circ} 2$.

Los análisis que se han realizado son frecuencias y pruebas de asociación (chi cuadrado y razón de probabilidades, OR) entre las variables del modelo, además de la construcción de un modelo logístico multivariado (stepwise), en el que también se ingresaron las variables que, aunque no siendo significativas en el análisis bivariado; por definición fueron importantes en explicar el marco conceptual de la desnutrición crónica, sin alterar la significancia del modelo. Es importante remarcar que el tipo de análisis que se está realizando en

${ }^{5}$ Utilizados en las publicaciones de estadísticas nacionales: INEI, Instituto Nacional de Salud (INS), etc. 
el presente trabajo es un análisis de muestras complejas, ya que el proceso de selección de la muestra no es aleatorio, por tanto, debe considerarse el efecto del diseño y el peso de ponderación al realizar el análisis correspondiente. En ambos casos, ya sea para el análisis bivariado, así como para el multivariado, se utilizó como nivel de significancia $0.10 \%$, asumiendo un rechazo o pérdida no mayor del $10 \%$, con un nivel de confianza del $95 \%$.

El programa estadístico utilizado para procesar la información del presente informe fue SPSS versión 13, que tiene el módulo de análisis de muestras complejas. Parte de los análisis, específicamente lo que corresponde al análisis multivariado, se realizaron en el programa STATA versión 8, ya que tiene mayor cantidad de análisis para muestras complejas.

Tabla 1. Variables del estudio según el marco conceptual de la UNICEF (1998)

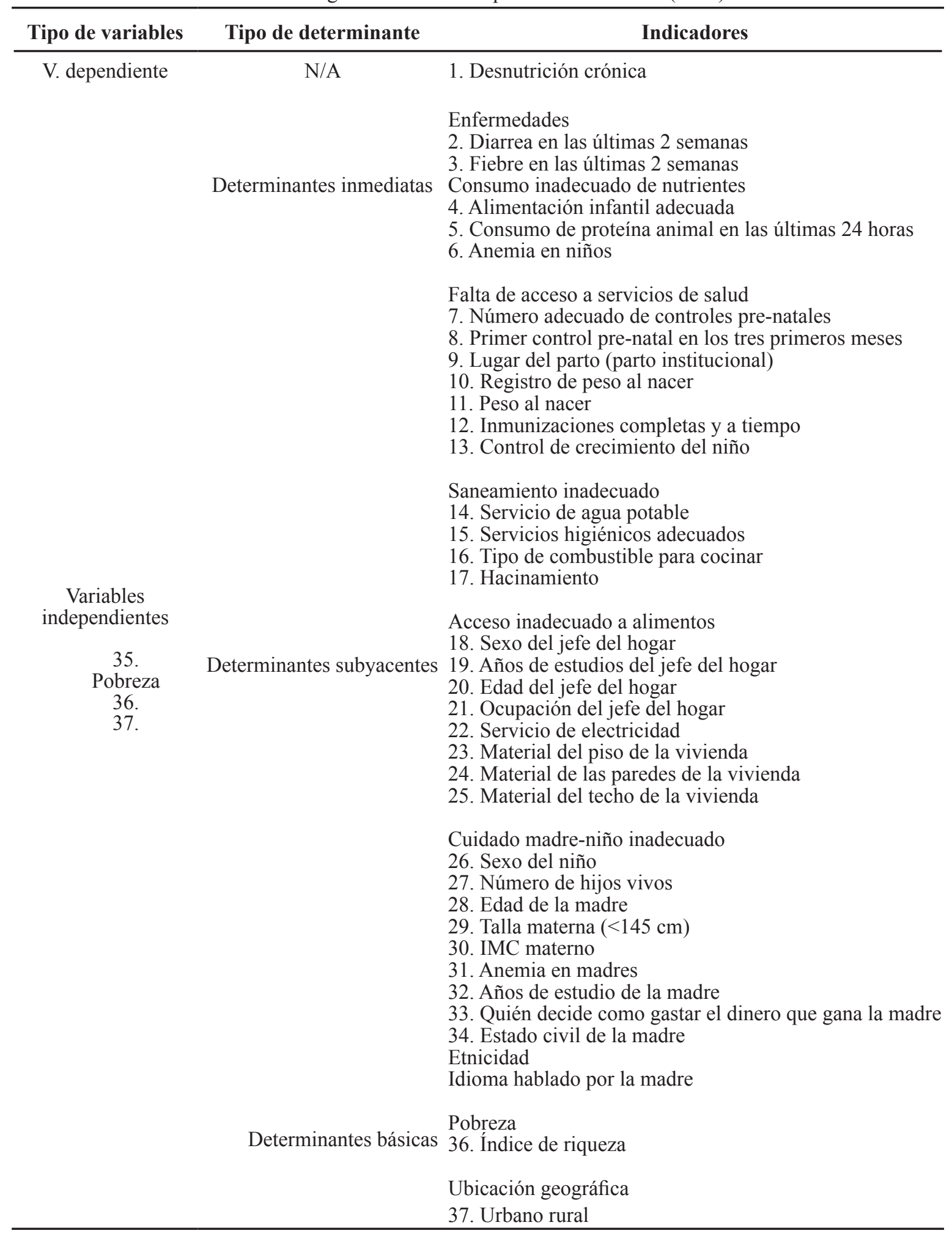


Tabla 2. Definición de las variables analizadas

\begin{tabular}{|c|c|c|}
\hline $\mathbf{N}^{\mathbf{0}}$ & Variable & Definición \\
\hline 1 & Desnutrición crónica & $\begin{array}{l}\text { Para evaluar el retardo de crecimiento en este grupo de edad, que es una forma de } \\
\text { desnutrición, se utiliza el indicador Talla para la Edad (T/E), y si es menor a -2 DE } \\
\text { con respecto al patrón de referencia (NCHS), se puede decir que el niño tiene retardo } \\
\text { de crecimiento, llamado en inglés stunting (OMS, 1983). }\end{array}$ \\
\hline 2 & $\begin{array}{l}\text { Enfermedades } \\
\text { diarreicas }\end{array}$ & $\begin{array}{l}\text { Este indicador está construido en base a los episodios de diarrea presentados por el } \\
\text { niño y reportados por la madre. }\end{array}$ \\
\hline 3 & $\begin{array}{l}\text { Enfermedades } \\
\text { respiratorias agudas }\end{array}$ & $\begin{array}{l}\text { Construido utilizando dos indicadores, incluye a los niños con fiebre en los últimos } \\
\text { quince días previos al día en que la madre es encuestada, lo cual es considerado que el } \\
\text { niño tiene una infección respiratoria aguda. }\end{array}$ \\
\hline 4 & $\begin{array}{l}\text { Alimentación } \\
\text { infantil adecuada }\end{array}$ & $\begin{array}{l}\text { La ENDES no recoge información de consumo del niño ni de la madre, pero se le } \\
\text { pregunta a la madre si el día anterior lo dio leche materna, además si consumió otros } \\
\text { alimentos. La alimentación adecuada está en función de la edad del niño, esto está en } \\
\text { relación con los Lineamientos de Nutrición Materno-Infantil (INS, 2003). } \\
\text { - Menores de } 6 \text { meses: Lactancia materna exclusiva } \\
\text { - De } 6 \text { a menos de } 9 \text { meses: Lactancia materna y dos a más raciones de alimentos } \\
\text { semisólidos o sólidos. } \\
\text { - De } 9 \text { a menores de } 12 \text { meses: Lactancia materna y tres a más raciones de alimentos } \\
\text { semisólidos o sólidos. } \\
\text { - De } 12 \text { meses a menores de } 24 \text { meses: Lactancia materna y cuatro a más raciones de } \\
\text { alimentos semisólidos o sólidos. } \\
\text { - De } 24 \text { meses a menores de } 36 \text { meses: Cinco a más raciones de alimentos semisólidos } \\
\text { o sólidos. }\end{array}$ \\
\hline 5 & $\begin{array}{l}\text { Consumo de } \\
\text { proteína animal en } \\
\text { las últimas } 24 \text { horas }\end{array}$ & $\begin{array}{l}\text { Al igual que los anteriores se construye con lo que la madre recuerda sobre la } \\
\text { alimentación del niño el día anterior, y es una variable de dos categorías que da } \\
\text { información acerca del consumo o no de algún alimento de origen animal. }\end{array}$ \\
\hline 6 & Anemia en niños & $\begin{array}{l}\text { Tomada a partir del recojo de hemoglobina, considerando la altura de la zona en la que } \\
\text { viven. A nivel del mar el punto de corte para considerar que un menor de } 36 \text { meses } \\
\text { tiene anemia es cuando su hemoglobina está por debajo de } 11 \mathrm{~g} / \mathrm{dL} \text {. En menores de } 6 \\
\text { meses no se puede considerar debido a que los puntos establecidos están dados para } \\
\text { mayores de } 6 \text { meses. (Bowman y Russell, 2003). }\end{array}$ \\
\hline 7 & $\begin{array}{l}\text { Número adecuado } \\
\text { de controles pre- } \\
\text { natales }\end{array}$ & $\begin{array}{l}\text { Según lo manejado tradicionalmente en MINSA, se asume como adecuado cuando la } \\
\text { gestante va un mínimo de seis controles pre-natales. }\end{array}$ \\
\hline 8 & $\begin{array}{l}\text { Primer Control pre- } \\
\text { natal en los primeros } \\
\text { tres meses }\end{array}$ & $\begin{array}{l}\text { Para considerar adecuado un control pre-natal para el presente estudio, la madre habrá } \\
\text { realizado sus controles en los primeros tres meses de gestación por lo menos una vez. }\end{array}$ \\
\hline 9 & Lugar del parto & $\begin{array}{l}\text { Lo que se trata de conocer con esta variable es la frecuencia de parto institucional. Esta } \\
\text { variable entra sin modificaciones y está definido como aquél que es atendido por un } \\
\text { profesional de la salud en un establecimiento de salud, que incluye hospitales, postas, } \\
\text { centros de salud y clínicas (MINSA). Se ha ingresado en dos categorías, definidas por } \\
\text { lo adecuado (en establecimientos de salud, cualquiera que sea) e inadecuado (en su } \\
\text { vivienda y otros lugares). }\end{array}$ \\
\hline 10 & $\begin{array}{l}\text { Registro de peso al } \\
\text { nacer }\end{array}$ & Variable que sirve para identificar si el niño fue o no pesado al momento de nacer. \\
\hline 11 & Peso al nacer & $\begin{array}{l}\text { Elaborada a partir del peso al nacer, y se categoriza en dos: bajo peso al nacer (peso } \\
\text { inferior a } 2500 \mathrm{~g} \text { ); y peso mayor o igual a } 2500 \mathrm{~g} \text {, que puede incluir además de los } \\
\text { niños que nacen con un peso adecuado, a los que son macrosómicos. Los niños que } \\
\text { nacen con un peso que no llega a ser considerado como bajo al nacer, están en la } \\
\text { categoría de peso insuficiente (peso al nacer de } 2500 \text { a } 2799 \mathrm{~g} \text { ) (OMS, 1995). }\end{array}$ \\
\hline
\end{tabular}


Continuación de Tabla 2

\begin{tabular}{|c|c|c|c|c|c|c|}
\hline \multirow{8}{*}{12} & \multirow{8}{*}{$\begin{array}{l}\text { Inmunizaciones } \\
\text { completas }\end{array}$} & \multicolumn{5}{|c|}{$\begin{array}{l}\text { El cual ha sido obtenido considerando las vacunas específicas (tales como la DTP, } \\
\text { Sarampión, BCG. Polio, etc.) de los niños de } 12 \text { a } 23 \text { meses. Este indicador no incluye } \\
\text { vacunas inespecíficas, que son las que la madre no recuerda. En el Perú desde el año } \\
\text { 2005, hay un nuevo esquema de vacunación el cual es: }\end{array}$} \\
\hline & & Grupo de edad & & \multicolumn{2}{|c|}{ Edad de aplicación } & Tipo de vacuna \\
\hline & & \multirow{4}{*}{\multicolumn{2}{|c|}{ Menores de un año }} & \multicolumn{2}{|c|}{ Recién nacido } & $\mathrm{BCG}+\mathrm{HvB}$ \\
\hline & & & & \multicolumn{2}{|c|}{2 meses } & PENTA + VOP \\
\hline & & & & \multicolumn{2}{|c|}{3 meses } & Tetra o DPT + Hib + VOP \\
\hline & & & & \multicolumn{2}{|c|}{4 meses } & PENTA + VOP \\
\hline & & \multicolumn{2}{|c|}{ De un año o mayores } & \multicolumn{2}{|c|}{12 meses } & SPR + AMA \\
\hline & & \multicolumn{5}{|c|}{ Fuente: Página web del Ministerio de Salud } \\
\hline \multirow{8}{*}{13} & \multirow{8}{*}{$\begin{array}{l}\text { Control de } \\
\text { crecimiento }\end{array}$} & \multicolumn{5}{|c|}{$\begin{array}{l}\text { Elaborado en base al número de veces que la madre llevó a su niño al establecimiento } \\
\text { de salud. Existe un esquema del MINSA, en el Manual de procedimientos para el } \\
\text { control del crecimiento y desarrollo de la niña y el niño: }\end{array}$} \\
\hline & & $\begin{array}{c}\text { GRUPO } \\
\text { ETAREO }\end{array}$ & $\mathrm{CO}$ & LES & & EADES \\
\hline & & Recién nacido & & & $\mathrm{Al} 2^{\circ}$ día del & ${ }^{\circ}$ día de nacido \\
\hline & & $<12$ meses & & & 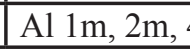 & $7 \mathrm{~m}$ y 9 meses edad \\
\hline & & De $1 \mathrm{a}<2$ años & & & $\mathrm{A} \operatorname{los} 12 \mathrm{~m}, 1$ & $\mathrm{~m}$ y $21 \mathrm{meses}$ edad \\
\hline & & De 2 a 4 años & & año & $\mathrm{A} \operatorname{los} 24 \mathrm{~m}, 3$ & m, $42 \mathrm{~m}$ y 48 meses edad \\
\hline & & De 5 a 9 años & & & $\mathrm{A} \operatorname{los} 5 \mathrm{a}, 6^{\mathrm{a}}$, & 9 años edad \\
\hline & & \multicolumn{5}{|c|}{$\begin{array}{l}\text { Fuente: MINSA (documento en consulta) http://www.minsa.gob.pe/portal/p2005/ } \\
\text { docconsulta.asp }\end{array}$} \\
\hline 14 & $\begin{array}{l}\text { Servicio de agua } \\
\text { potable }\end{array}$ & \multicolumn{5}{|c|}{ Indica qué viviendas cuentan con servicio de agua potable dentro y fuera de la misma. } \\
\hline 15 & $\begin{array}{l}\text { Servicios higiénicos } \\
\text { adecuado }\end{array}$ & \multicolumn{5}{|c|}{$\begin{array}{l}\text { Construida como una variable dicotómica, separa a las viviendas que cuentan con red } \\
\text { pública dentro y fuera de su vivienda, además de letrinas, siendo todos estos tipos } \\
\text { de servicios higiénicos considerados como adecuados; mientras que están dentro de } \\
\text { inadecuado las viviendas que no cuentan con servicio higiénico, por ejemplo. }\end{array}$} \\
\hline 16 & $\begin{array}{l}\text { Tipo de combustible } \\
\text { para cocinar }\end{array}$ & \multicolumn{5}{|c|}{$\begin{array}{l}\text { Están considerados como adecuados (como electricidad y gas) los que no producen } \\
\text { emisiones de humo, que por lo general puedan contaminar el interior de las viviendas } \\
\text { de las familias encuestadas. }\end{array}$} \\
\hline 17 & Hacinamiento & \multicolumn{5}{|c|}{$\begin{array}{l}\text { Esta variable se ha construido a partir de dos más, es decir que la variable hacinamiento } \\
\text { es la relación existente entre el número de habitaciones entre el número de miembros } \\
\text { del hogar. Si este número obtenido de la división es mayor o igual a tres, el hogar es } \\
\text { considerado como hacinado. }\end{array}$} \\
\hline 18 & $\begin{array}{l}\text { Sexo del jefe del } \\
\text { hogar }\end{array}$ & \multicolumn{5}{|c|}{$\begin{array}{l}\text { El jefe del hogar es a quien la persona que responde la encuesta considera como tal, } \\
\text { según su propio criterio. En este caso, la persona que responde el cuestionario es la } \\
\text { madre del niño. }\end{array}$} \\
\hline 19 & $\begin{array}{l}\text { Años de estudio del } \\
\text { jefe del hogar }\end{array}$ & \multicolumn{5}{|c|}{$\begin{array}{l}\text { Variable que nos da idea del logro académico alcanzado por el jefe del hogar, } \\
\text { siendo considerado por años de estudios, ya que como años de estudios brinda más } \\
\text { información, al mismo tiempo de que sea comparable con estudios fuera del Perú. Las } \\
\text { dos categorías son: De } 7 \text { a } 17 \text {, y menos de } 6 \text { años de estudios. }\end{array}$} \\
\hline 20 & $\begin{array}{l}\text { Edad del jefe del } \\
\text { hogar }\end{array}$ & \multicolumn{5}{|c|}{$\begin{array}{l}\text { Colocado en grupos de edad: Mayor o igual a } 45 \text { años y la otra categoría que es menor } \\
\text { de } 45 \text { años de edad. }\end{array}$} \\
\hline 21 & $\begin{array}{l}\text { Ocupación del jefe } \\
\text { del hogar }\end{array}$ & \multicolumn{5}{|c|}{$\begin{array}{l}\text { Indicador que tiene dos categorías: "trabajó"y "no trabajó" que se refiere a la presencia } \\
\text { o ausencia de una actividad económica dentro o fuera del hogar en la semana anterior. }\end{array}$} \\
\hline
\end{tabular}


DETERMINANTES DE LA DESNUTRICIÓN CRÓNICA DE LOS MENORES DE TRES AÑOS EN LAS REGIONES DEL PERÚ: SUBANÁLISIS DE LA ENCUESTA ENDES 2000

Julio - Diciembre 2016

\begin{tabular}{|c|c|c|}
\hline 22 & $\begin{array}{l}\text { Servicio de } \\
\text { electricidad }\end{array}$ & $\begin{array}{l}\text { Identifica los individuos que habitan en viviendas que cuentan con el servicio de } \\
\text { electricidad. }\end{array}$ \\
\hline 23 & Material del piso & Considera el tipo predominante de material del piso de la vivienda. \\
\hline 24 & $\begin{array}{l}\text { Material de las } \\
\text { paredes }\end{array}$ & $\begin{array}{l}\text { Variable que recoge información sobre el material predominante de las paredes de la } \\
\text { vivienda del niño y su madre. }\end{array}$ \\
\hline 25 & Material del techo & Brinda información sobre el material predominante del techo de la vivienda. \\
\hline 26 & Sexo del niño & Masculino o femenino. \\
\hline 27 & $\begin{array}{l}\text { Número de hijos } \\
\text { vivos }\end{array}$ & Variable que considera el número de hijos que viven en el hogar. \\
\hline 28 & Edad de la madre & $\begin{array}{l}\text { Variable originalmente continua, se categoriza en grupos de edades: menores de } 35 \\
\text { años y de } 35 \text { a más. }\end{array}$ \\
\hline 29 & Talla materna & Dos categorías: menor a $145 \mathrm{~cm}$, o mayor o igual a ese punto de corte. \\
\hline 30 & IMC materno & Tiene tres categorías: Normal, sobrepeso y bajo peso. \\
\hline 31 & Anemia en madres & Está tal cual es construida por INEI (presencia o ausencia de anemia). \\
\hline 32 & $\begin{array}{l}\text { Años de estudios de } \\
\text { la madre }\end{array}$ & $\begin{array}{l}\text { Considerado por años de estudios, tiene dos categorías: menos de } 9 \text { años de estudios, } \\
\text { y de } 9 \text { a más años en aulas. }\end{array}$ \\
\hline 33 & $\begin{array}{l}\text { Quien decide cómo } \\
\text { gastar el dinero de la } \\
\text { madre }\end{array}$ & $\begin{array}{l}\text { Dos categorías: una en la que ella decide sola o con otros, y otra en la que ella no } \\
\text { decide. }\end{array}$ \\
\hline 34 & $\begin{array}{l}\text { Estado civil de la } \\
\text { madre }\end{array}$ & Sin pareja o con pareja (dos categorías). \\
\hline 35 & $\begin{array}{l}\text { Idioma hablado por } \\
\text { la madre }\end{array}$ & $\begin{array}{l}\text { Construida a partir de la información que recoge la encuesta ENDES, esta variable } \\
\text { divide a las madres en dos categorías que son madres que hablan castellano y las que } \\
\text { hablan otro idioma, sea este quechua, aymara u otro dialecto. }\end{array}$ \\
\hline 36 & Índice de riqueza & $\begin{array}{l}\text { El índice de riqueza o wealth index es utilizado para construir esta variable y se crea } \\
\text { a través de un análisis de componentes principales, en los que entran variables como } \\
\text { servicio de agua, servicios higiénicos, posesión de bienes físicos, entre otros y en el } \\
\text { cual se obtiene un puntaje. }\end{array}$ \\
\hline 37 & Lugar de residencia & $\begin{array}{l}\text { Esta variable divide a la población estudiada en dos de acuerdo a la zona de residencia } \\
\text { de la familia, ya sea zona urbana o rural. }\end{array}$ \\
\hline
\end{tabular}

\section{Resultados y discusión}

\subsection{Desnutrición crónica}

La desnutrición crónica está en el $21.7 \%$ de los menores de 3 años, mientras que entre los menores de 5 es $25.4 \%$ (INEI, 2001).

Tabla 3. Estado nutricional de los menores de tres años, según grupos de edad.

\begin{tabular}{cc}
\hline $\begin{array}{c}\text { Grupos de edad en } \\
\text { meses }\end{array}$ & $\begin{array}{c}\text { Porcentaje de } \\
\text { desnutrición }(\mathbf{N})\end{array}$ \\
\hline$<6$ & $3.9(854)$ \\
$6 \mathrm{a}<12$ & $11.1(981)$ \\
$12 \mathrm{a}<24$ & $28.3(2150)$ \\
$24 \mathrm{a}<36$ & $27.2(2084)$ \\
\hline Total & $\mathbf{2 1 . 7 ( 6 0 6 9})$ \\
\hline
\end{tabular}

Se puede observar que el grupo que tiene mayor prevalencia de desnutrición crónica declina a medida que aumenta la edad luego de los 24 meses.
Tabla 4. Estado nutricional de los menores de tres años, clasificados según ámbitos, ENDES 2000.

\begin{tabular}{cc}
\hline Ámbito & $\begin{array}{c}\text { Porcentaje de } \\
\text { desnutrición (N) }\end{array}$ \\
\hline Lima Metropolitana & $6.2(1357)$ \\
Resto Costa & $13.7(1321)$ \\
Sierra Urbana & $17.9(650)$ \\
Sierra Rural & $39.3(1800)$ \\
Selva & $24.2(943)$ \\
\hline Total & $\mathbf{2 1 . 7 ( 6 0 7 0 )}$ \\
\hline
\end{tabular}

La mayor parte de los menores de tres años con desnutrición crónica están en la Sierra Rural. Por otro lado, la menor cantidad de prevalencia de retardo del crecimiento se da en Lima, con tan sólo 6.2\%; pero es importante recordar que, aunque el porcentaje es pequeño, en Lima Metropolitana se encuentra casi la tercera parte de la población del país (INEI, 2008). 


\subsection{Análisis multivariado de los determinantes}

\subsubsection{En Lima Metropolitana}

Los determinantes de la desnutrición identificados son los años de estudios de la madre del menor $(p=0.087)$, el número de controles pre-natales ${ }^{6}(p=0.008)$, así como el pertenecer a los quintiles inferiores de pobreza $(p=0.087)$, el bajo peso al nacer $(p=0.391)$ y la ausencia de servicios higiénicos en la vivienda $(p=0.920)$. La variable de bajo peso al nacer tiene un OR ajustado de 5.66 lo que significa que los niños con un bajo peso tienen casi seis veces la probabilidad de tener desnutrición.

Tabla 5. Factores asociados a la desnutrición crónica en niños menores de tres años en Lima Metropolitana, ENDES 2000.

\begin{tabular}{lccc}
\hline \multicolumn{1}{c}{ Variable } & $\begin{array}{c}\text { OR } \\
\text { Bivariado } \\
(\mathbf{N C}-\mathbf{9 5 \%})\end{array}$ & $\begin{array}{c}\text { OR } \\
\text { Ajustado } \\
(\mathbf{N C}-\mathbf{9 5 \%})\end{array}$ & $\boldsymbol{p}$ \\
\hline $\begin{array}{l}\text { Años de estudio de } \\
\text { la madre } \\
\quad 8 \text { años ó menos }\end{array}$ & 3.84 & 1.89 & 0.087 \\
$\begin{array}{l}\text { Número de } \\
\text { controles prenatales } \\
<6 \text { controles } \\
\text { Quintiles de } \\
\text { pobreza }\end{array}$ & 3.98 & 2.97 & 0.008 \\
$\begin{array}{l}\text { Quintil II y III } \\
\text { Bajo peso al nacer } \\
<2.5 \text { Kg }\end{array}$ & 3.09 & 2.25 & 0.087 \\
$\begin{array}{l}\text { Servicios higiénicos } \\
\text { Sin servicio higiénico }\end{array}$ & 2.25 & 5.62 & 0.391 \\
\hline
\end{tabular}

Los menores de tres años tienen el doble de riesgo de tener desnutrición crónica cuando la madre tiene menos de nueve años de estudio $(\mathrm{OR}=1.89 ; p=0.087)$ o cuando pertenece a un hogar del quintil I y II $(\mathrm{OR}=2.25 ; p=0.087)$. Algo similar se ha reportado en Guatemala, los niños de madres analfabetas fueron cinco $(\mathrm{OR}=5,0$; IC 95\%: 1,35; 16,67) veces más desnutridos comparados con sus contrapartes hijos de madres que saben leer y escribir (Sereebutra et al, 2006).

La falta de servicios higiénicos no es una variable significativa, pero tampoco le resta significancia al modelo, además no hay un mayor riesgo de desnutrición crónica en los menores que presenten esta característica $(\mathrm{OR}=1.09 ; p=0.92)$.

\subsubsection{En el Resto de Costa}

En este ámbito resultaron más notables los determinantes que corresponden al acceso a servicios de salud como bajo peso al nacer $(\mathrm{OR}=2.89 ; p=0.002)$, y controles de crecimiento y desarrollo (CRED) al día con casi el doble de probabilidades de ser desnutrido cuando no cumplieron el esquema $(\mathrm{OR}=1.81 ; p=0.043)$.

${ }^{6}$ Es 3 veces más probable ser desnutrido cuando se tiene menos de 6 controles prenatales.
Tabla 6. Factores asociados a la desnutrición crónica en niños menores de tres años en Resto Costa, ENDES 2000.

\begin{tabular}{|c|c|c|c|}
\hline Variable & $\begin{array}{c}\text { OR Bivariado } \\
(\mathrm{NC}-95 \%)\end{array}$ & $\begin{array}{c}\text { OR } \\
\text { Ajustado } \\
(\mathrm{NC}-\mathbf{9 5 \%})\end{array}$ & $p$ \\
\hline $\begin{array}{l}\text { Tiene servicio de } \\
\text { electricidad }\end{array}$ & & & \\
\hline $\begin{array}{l}\text { No } \\
\text { Bajo peso al } \\
\text { nacer }\end{array}$ & 2.91 & 1.76 & 0.074 \\
\hline $\begin{array}{l}<2.5 \mathrm{Kg} \\
\text { Controles CRED } \\
\text { al día }\end{array}$ & 3.00 & 2.89 & 0.002 \\
\hline $\begin{array}{l}\text { No } \\
\text { Talla de la madre } \\
<145 \mathrm{~cm}\end{array}$ & 1.93 & 1.81 & 0.043 \\
\hline $\begin{array}{l}\mathrm{Si} \\
\text { Número de hijos } \\
\text { vivos }\end{array}$ & 2.17 & 1.94 & 0.044 \\
\hline $\begin{array}{l}3 \text { ó más } \\
\text { Años de estudio } \\
\text { de la madre }\end{array}$ & 2.32 & 1.64 & 0.035 \\
\hline 4 ó menos años & 2.83 & 1.42 & 0.291 \\
\hline
\end{tabular}

La talla materna menor a $145 \mathrm{~cm}$ resultó estar asociada a la desnutrición crónica de sus hijos ( $\mathrm{OR}=1.94$; $\mathrm{p}=0.044)$, así como los años de estudio de la madre, con un riesgo de desnutrición de su hijo cuando la madre tiene 4 ó menos años de estudio $(\mathrm{OR}=1.42 ; p=0.291)$; y el tener 3 ó más hijos $(\mathrm{OR}=1.64 ; p=0.035)$. Finalmente, los hogares $\sin$ electricidad tuvieron un mayor porcentaje de desnutridos, siendo el riesgo de 1.76 veces mayor que en los que sí contaban con fluido eléctrico $(\mathrm{OR}=1.76 ; p=0.074)$.

\subsubsection{En la Sierra Urbana}

Al igual que en el Resto de Costa, en la Sierra Urbana, los factores más asociados a la desnutrición crónica han sido los que tienen que ver con los servicios de salud.

Tabla 7. Factores asociados a la desnutrición crónica en niños menores de tres años en Sierra Urbana, ENDES 2000 .

\begin{tabular}{lccc}
\hline \multicolumn{1}{c}{ Variable } & $\begin{array}{c}\text { OR } \\
\text { Bivariado } \\
(\mathbf{N C}-\mathbf{9 5 \%})\end{array}$ & $\begin{array}{c}\text { OR } \\
\text { Ajustado } \\
(\mathbf{N C}-\mathbf{9 5 \%})\end{array}$ & $\boldsymbol{p}$ \\
\hline $\begin{array}{l}\text { Tiene servicio de } \\
\text { electricidad } \\
\text { No }\end{array}$ & 4.05 & 2.16 & 0.055 \\
$\begin{array}{l}\text { Bajo peso al nacer } \\
<2.5 \mathrm{Kg}\end{array}$ & 5.3 & 4.62 & 0.001 \\
$\begin{array}{l}\text { Controles CRED al día } \\
\text { No }\end{array}$ & 2.63 & 2.69 & 0.008 \\
$\begin{array}{l}\text { Parto Institucional } \\
\text { No }\end{array}$ & 2.76 & 2.12 & 0.009 \\
\hline
\end{tabular}

El bajo peso al nacer demostró ser un fuerte predictor de la desnutrición ya que presentan un riesgo de más 
de cuatro veces los niños que han nacido con un peso menor a $2.5 \mathrm{Kg}(\mathrm{OR}=4.62 ; p=0.055)$; mientras que la ausencia del parto institucional $(\mathrm{OR}=2.12 ; p=0.009)$, de controles CRED $(\mathrm{OR}=2.69 ; p=0.008)$ significaron el doble de probabilidades de que el niño presente retardo de crecimiento.

La falta de servicio eléctrico en la vivienda está asociado a la desnutrición crónica, trayendo el doble de riesgo en los menores que las habitan $(\mathrm{OR}=2.16 ; p=0.055)$.

\subsubsection{En la Sierra Rural}

Los pocos años de estudio de la madre $^{7}(\mathrm{OR}=1.67$; $p=0.003)$, la baja talla de la madre ${ }^{8}(\mathrm{OR}=2.36 ; \mathrm{p}=0.000)$, la ausencia de parto institucional $(\mathrm{OR}=1.75 ; p=0.002)$, 6 ó menos controles prenatales ( $\mathrm{OR}=1.39 ; \mathrm{p}=0.013)$; 3 ó más hijos vivos $(\mathrm{OR}=1.43 ; p=0.002)$; y el idioma materno $^{9}$ diferente al castellano $(\mathrm{OR}=1.29 ; p=0.041)$; fueron las más relacionadas con la desnutrición crónica en los menores de tres años.

Tabla 8. Factores asociados a la desnutrición crónica en niños menores de tres años en Sierra Rural, ENDES 2000.

\begin{tabular}{|c|c|c|c|}
\hline Variable & $\begin{array}{c}\text { OR } \\
\text { Bivariado } \\
(\mathrm{NC}-\mathbf{9 5 \%})\end{array}$ & $\begin{array}{c}\text { OR } \\
\text { Ajustado } \\
(\mathrm{NC}-\mathbf{9 5 \%})\end{array}$ & $p$ \\
\hline \multicolumn{4}{|l|}{$\begin{array}{l}\text { Años de estudio de } \\
\text { la madre }\end{array}$} \\
\hline$<8$ años & 2.66 & 1.67 & 0.003 \\
\hline $\begin{array}{l}\text { Talla de la madre } \\
\text { menor a } 145 \mathrm{~cm} .\end{array}$ & & & \\
\hline Sí & 2.47 & 2.36 & 0.000 \\
\hline Parto institucional & 57 & 175 & \\
\hline \multicolumn{4}{|l|}{$\begin{array}{l}\text { Controles CRED al } \\
\text { día }\end{array}$} \\
\hline No & 1.71 & 1.53 & 0.002 \\
\hline Controles prenatales & & & \\
\hline \multicolumn{4}{|l|}{ Servicios higiénicos } \\
\hline $\begin{array}{l}\text { Sin servicio/Río } \\
\text { acequia o canal }\end{array}$ & 1.41 & 1.29 & 0.030 \\
\hline $\begin{array}{l}\text { Número de hijos } \\
\text { vivos }\end{array}$ & & & \\
\hline 3 ó más & 1.96 & 1.43 & 0.002 \\
\hline \multicolumn{4}{|l|}{$\begin{array}{l}\text { Idioma hablado por } \\
\text { la madre }\end{array}$} \\
\hline Otro & 1.45 & 1.29 & 0.041 \\
\hline
\end{tabular}

La ausencia de control de crecimiento y desarrollo (CRED) en niños constituyó un riesgo de 1.5 veces más de presentar desnutrición crónica $(\mathrm{OR}=1.53 ; p=0.002)$. La probabilidad de desnutrición fue 1.3 veces más en menores que vivían en hogares sin servicios higiénicos, en comparación con los que sí tuvieron $(\mathrm{OR}=1.29 ; p=0.030)$.

\subsubsection{En la Selva}

Para este ámbito fueron también muy notables las ${ }^{7} 8$ ó menos.

${ }^{8}$ Menor a $145 \mathrm{~cm}$.

${ }^{9}$ En la Sierra Rural, en donde hay $47.2 \%$ de madres de los menores de tres años que hablan un idioma diferente al castellano (principalmente quechua o aymara). características maternas, resultando que, si la madre tiene 4 ó más hijos, hubo casi el triple $(\mathrm{OR}=2.89, p=0.005) \mathrm{de}$ riesgo de tener un hijo con desnutrición crónica.

Tabla 9. Factores asociados a la desnutrición crónica en niños menores de tres años en Selva, ENDES 2000.

\begin{tabular}{|c|c|c|c|}
\hline Variable & $\begin{array}{c}\text { OR } \\
\text { Bivariado } \\
(\mathrm{NC}-\mathbf{9 5 \%})\end{array}$ & $\begin{array}{c}\text { OR Ajustado } \\
(\mathrm{NC}-95 \%)\end{array}$ & $p$ \\
\hline $\begin{array}{l}\text { Años de estudio de } \\
\text { la madre }\end{array}$ & & & \\
\hline 4 ó más & 2.12 & 2.89 & 0.005 \\
\hline $\begin{array}{l}\text { Otro idioma } \\
\text { Otaure }\end{array}$ & 2.59 & 1.81 & 0.042 \\
\hline $\begin{array}{l}\text { Parto institucional } \\
\text { No }\end{array}$ & 2.49 & 1.94 & 0.029 \\
\hline $\begin{array}{l}\text { Controles } \\
\text { prenatales }\end{array}$ & & & \\
\hline $\begin{array}{l}\text { Menos de } 6 \\
\text { Controles CRED } \\
\text { al día }\end{array}$ & 2.65 & 1.64 & 0.014 \\
\hline No & 2.21 & 1.42 & 0.53 \\
\hline
\end{tabular}

Los niños están más expuestos a problemas de desnutrición crónica si sus madres estudiaron menos de nueve años de en aulas $(\mathrm{OR}=1.76 ; p=0.069)$; tienen como idioma materno otro diferente al castellano $(\mathrm{OR}=1.81$; $p=0.042)$; y carecen de parto institucional $(\mathrm{OR}=1.94$; $p=0.029$ ). Si la madre durante la gestación tuvo menos de seis controles pre-natales, tiene 1.6 veces más de tener un desnutrido $(\mathrm{OR}=1.64 ; p=0.014)$. Finalmente, se incluyó la variable de controles CRED la cual no es significativa, pero tampoco le resta significancia al modelo multivariado para la Selva.

Resumiendo, en la tabla siguiente se han ordenado los determinantes de la desnutrición crónica en orden de importancia, siendo los más importantes los que más se han presentado en las zonas analizadas, mostrándose que los factores que mayor asociación han presentado con el retardo de crecimiento, son aquellos que corresponden a la situación y condiciones de salud de la mujer. 
Tabla 10. Resumen de determinantes de desnutrición crónica, considerando en quiénes se toman las acciones y qué tipo

\begin{tabular}{|c|c|c|c|}
\hline $\begin{array}{l}\text { Determinantes asociados a la } \\
\text { desnutrición crónica }\end{array}$ & $\begin{array}{c}\text { Número de modelos } \\
\begin{array}{c}\text { que incluyen los } \\
\text { determinantes }\end{array}\end{array}$ & $\begin{array}{c}\text { Acciones en público } \\
\text { objetivo }\end{array}$ & Tipo de acciones a tomar \\
\hline Años de estudio de la madre & 4 & \multirow{2}{*}{$\begin{array}{l}\text { Mujeres en edad fértil } \\
\text { Niños }\end{array}$} & \multirow{2}{*}{$\begin{array}{l}\text { Situación de la mujer } \\
\text { Condiciones de salud }\end{array}$} \\
\hline $\begin{array}{l}\text { Controles de crecimiento y } \\
\text { desarrollo (CRED) }\end{array}$ & 4 & & \\
\hline Controles pre-natales & 3 & Mujeres en edad fértil & Condiciones de salud \\
\hline Número de hijos & 3 & Mujeres en edad fértil & Situación de la mujer \\
\hline Lugar del parto & 3 & Mujeres en edad fértil & Condiciones de salud \\
\hline Bajo peso al nacer & 3 & Mujeres en edad fértil & Condiciones de salud \\
\hline Talla materna & 2 & Mujeres en edad fértil & Condiciones de salud \\
\hline Idioma de la madre & 2 & Mujeres en edad fértil & Situación de la mujer \\
\hline Servicio de electricidad en hogar & 2 & Mujeres en edad fértil & Situación de la mujer \\
\hline Servicios higiénicos en hogar & 2 & Mujeres en edad fértil & Situación de la mujer \\
\hline Quintil de ingreso & 1 & Mujeres en edad fértil & Situación de la mujer \\
\hline
\end{tabular}

\section{Conclusiones}

1. Los datos han demostrado que lo más importante para reducir las tasas de desnutrición crónica en menores de tres años en los diferentes ámbitos del Perú, es tomar acciones en las mujeres (madres), mejorando su situación y el acceso a los servicios de salud (condiciones de salud).

2. Los factores asociados más importantes en todos los ámbitos fueron: educación de la madre, controles de crecimiento del niño, número de controles pre-natales adecuados, parto institucional, peso al nacer y número de hijos vivos.

3. Los programas y proyectos orientados a reducir la desnutrición crónica deben enfatizar las acciones sobre la mujer, ya que podrían mejorar los resultados obtenidos.

\section{Agradecimientos}

Se agradece la colaboración de Carlos E. Rojas, funcionario de CARE Perú, ya que los análisis se hicieron en el marco de un proyecto en curso de dicha ONG.

\section{Literatura citada}

ACC/SCN. 2001. What works? A review of the efficacy and effectiveness of nutrition intervention, Allen LH, Gillespie SR. ACC/SCN: Geneva in collaboration with the Asian Development Bank, Manila. 124 p.

Arifeen, S. y Black, R. 2001. Exclusive Breastfeeding Reduces Acute Respiratory Infection and Diarrhea Deaths Among Infants in Dhaka Slums. Pediatrics 108(4):67-74.

Assis, A., Barreto, M., Santos, L., Fiaccone, R. y da Silva Gomes, G. 2005. Growth faltering in childhood related to diarrhea: a longitudinal community based study. Eur J Clin Nutr. 59:1317-23.

Bellessa, M., Forste, R. Haas, D. 2005. Maternal education and child nutritional status in Bolivia:finding the links. Soc Sci Med. 60: 395-407.

Bhandari, R. y Smith, F. 2000. Education and food consumption in China: household analysis and policy implications. J Nutr Educ. 32:214-24.
Bowman, B. y Russell, R. 2003. Conocimientos actuales de nutrición. Octava edición. Publicación Científica y Técnica No 592. Organización Panamericana de la Salud (OPS) / Organización Mundial de la Salud (OMS). Washington, D.C.

Brown, K., Black, R. and Becker S. 1982. Seasonal changes in nutritional status and the prevalence of malnutrition in a longitudinal study of young children in rural Bangladesh. Am J Clin Nutr. 36:303-13.

Brown, K. 2003. Diarrhea and Malnutrition. J. Nutr. 133:328S-32S.

Checkley, W. 2004. Effect of water and sanitation on childhood health in a poor peruvian peri-urban community. Lancet. 363:112-8.

De Caballero, E., Sinisterra, O., Lagrutta, F. y Atalah, E. 2004. Evaluación del impacto nutricional del programa de alimentación complementaria de Panamá en niños menores de 5 años. Arch Latinoam Nutr. 54(1):66-71.

Delpeuch, F., Traissac, P., Martin-Prével, M., Massamba, JP. and Maire, B. 2000. Economic crisis and malnutrition: socioeconomic determinants of anthropometric status of preschool children and their mothers in an African urban area. Public Health Nutr. 3(1):39-47.

Fewtrell, L., Kaufmann, R., Kay, D., Enanoria, W., Haller, L. and Colford, Jr. J. 2005. Water, sanitation, and hygiene interventions to reduce diarrhoea in less developed countries: a systematic review and metaanalysis. Lancet Infect Dis. 5:42-52.

Instituto Nacional de Estadística e Informática (INEI). 2001. Encuesta Demográfica y de Salud Familiar 2000 (ENDES 2000). Informe principal. Lima, Perú.

Instituto Nacional de Estadística e Informática (INEI). 2011. Perú: Indicadores de Resultados de los Programas Estratégicos, 2010. Encuesta Demográfica y de Salud Familiar - ENDES Continua (Primeros resultados). Lima, Perú.

Instituto Nacional de Estadística e Informática (INEI). 2008. Perú: crecimiento y distribución de la población 2007. Primeros resultados. Censos Nacionales 2007: XI de Población y VI de Vivienda. Lima, Perú. 
Instituto Nacional de Salud (INS) / Centro Nacional de Alimentación y Nutrición (CENAN). 2003. Lineamientos de Nutrición materno infantil. Lima, Perú.

Jukes, M. 2005. The long-term impact of preschool health and nutrition on education. Food and Nutrition Bulletin, 26 (2): S193-201.

Larrea, C., Montalvo, P. and Ricaurte, A. 2004. Child Malnutrition, Social Development and Health Services in the Andean Region. Interamerican Development Bank Flacso, Ecuador.

Lutter, C., Habicht, JP., Rivera, J, and Martorell, R. 1992. The relationship between energy intake and diarrhoeal disease in their effects on child growth: biological model, evidence, and implications for public health policy. Food Nutr Bull 14(1):36-42.

Marquis, G., Habicht, JP., Lanata, C., Black, R. and Rasmussen K. 1997. Association of Breastfeeding and Stunting in Peruvian Toddlers: An Example of Reverse Causality. Int J Epidemiol. 26:349-56.

Méndez, M. and Adair L. 1999. Severity and Timing of Stunting in the First Two Years of Life Affect Performance on Cognitive Tests in Late Childhood. J Nutr. 129:155562.

Merchant, AT., Jone,s C., Kiure, A., Kupka, R., Fitzmaurice, G., Herrera, MG. et al. 2003. Water and sanitation associated with improved child growth. Eur J Clin Nutr.; 57:1562-8.

Onyango, A., Koskia, K. and Tucke, K. 1998. Food diversity versus breastfeeding choice in determining anthropometric status in rural Kenyan toddlers. Int $\mathbf{J}$ Epidemiol. 27:484-89.

Organización Mundial de la Salud (OMS). 1995. El estado Físico, Uso e Interpretación de la Antropometría, Informe de un comité de expertos de la OMS. Serie de informes técnicos $\mathrm{N}^{\circ} 854$. Ginebra.

Organización Mundial de la Salud, (OMS). 1983. Medición del Cambio del Estado Nutricional. Ginebra.

Reyes, H., Pérez-Cuevas, R., Sandoval, A., Castillo, R., Santos, J., Doubova, S. and Gutiérrez G. 2004. The family as a determinant of stunting in children living in conditions of extreme poverty: a case-control study. BMC Public Health 4:57

Rice, A., Sacco, L., Hyder, A. and Black R. 2000. Malnutrition as an underlying cause of childhood deaths associated with infectious diseases in developing countries. Bull World Health Organ. 78:1207-21.

Ruel, M., Levin, C., Armar-Klemesu, M., Maxwell, D. and Morris, S. 1999. Good care practices can mitigate the negative Effects of poverty and low maternal schooling on Children's nutritional status: evidence from Accra. FCND Discussion Paper No 62. International Food Policy Research Institute. Washington D.C.
Sereebutra, P., Solomons, N., Aliyu, M and Jolly, P. 2006. Sociodemographic and environmental predictors of childhood stunting in rural Guatemala. Nutr Res. 26(2):65-70.

Smith, L., Haddad, L. 1990. Explaining child malnutrition in developing countries: a cross-country analysis. FCND Discussion Paper No. 60: International Food Policy Research Institute, Washington D.C. 1990.

Smith, L. and Haddad, L. 2002. How potent is economic growth in reducing undernutrition? What are the pathways of impact? New cross-country evidence. Economic Development and Cultural Change. 51, 1; ABI/INFORM Global pg. 55.

Stephensen, B. 1999. Burden of Infection on Growth Failure. J Nutr. 129(2S Suppl):534S-8S.

Suárez, M., Cuba, C., Reyes, W., Mendoza, E. y Salinas, J. 1999. Determinantes de la desnutrición aguda y crónica en niños menores de 3 años: Un subanálisis de la ENDES 1992 y 1996. Instituto Nacional de Estadística e Informática (INEI) y PRISMA - Dirección de Investigación. Lima, Perú. 59 p.

Thi Hop, L., Gross, R., Giay, T., Sastroamidjojo, S., Schultink, W. y Thi Lang, N. 2000. Premature Complementary Feeding Is Associated with Poorer Growth of Vietnamese Children. J. Nutr. 130:2683-90.

Ukwuani, F. and Suchindran, C. 2003. Implications of women's work for child nutritional status in sub-Saharan Africa: a case study of Nigeria. Soc Sci Med 56:2109-21.

United Nations Administrative Committee on Coordination Sub-Committee on Nutrition (ACC/ SCN). 2000. Fourth report on the World nutrition situation: nutrition throught the life cycle. Geneva.

United Nations Children's Fund (UNICEF). 1998. The state of the world's children.

United Nations Children's Fund (UNICEF), United States Agency for International Development (USAID). Buen Inicio Supervivencia, crecimiento y desarrollo temprano. 2005.

United Nations System, Standing Committee on Nutrition (SCN). 2005. Fifth report on the World nutrition situation: nutrition for improves development outcomes. Geneva.

VanDerslice, J., Popkin, B. and Briscoe J. 1994. Drinking-water quality, sanitation, and breast-feeding: their interactive effects on infant health. Bull World Health Organ. 72(4):589-601.

Villalpando, S. and Lopez-Alarcón, M. 2000. Growth Faltering Is Prevented by Breast-Feeding in Underprivileged Infants from Mexico City. J. Nutr. 130: 546-52. 